\title{
The unusual history of stroke due to coagulopathy caused by SARS-CoV-2 infection in a 14-year-old boy with two heart tumors
}

\author{
Lesław Szydłowski' , Katarzyna Gruszczyńska², Jacek Kusa' ${ }^{1}$ Piotr Stanek³, \\ Magdalena Machnikowska-Sokołowska², Aleksandra Morka, Grzegorz Zalewskii ${ }^{3}$ Ewa Moric-Janiszewska ${ }^{5}$, \\ Zbigniew Olczak ${ }^{6}$, Jerzy Pietruszewski , Piotr Palen' ${ }^{8}$, Justyna Poprocka ${ }^{7}$, Anetta Undas ${ }^{9}, 10$
}

\begin{abstract}
'Department of Pediatric Cardiology, Faculty of Medical Sciences, Medical University of Silesia in Katowice, Katowice, Poland
2Division of Diagnostic Imaging, Department of Radiology and Nuclear Medicine, Faculty of Medical Sciences in Katowice, Medical University of Silesia, Katowice, Poland

${ }^{3}$ Department of Pediatric Cardiac Surgery, John Paul II Upper Silesian Child Health Center in Katowice, Katowice, Poland

${ }^{4}$ Department of Pediatric Cardiosurgery and Cardiosurgical Intensive Care, University Children's Hospital, Faculty of Health Sciences, Jagiellonian University Medical College, Kraków, Poland

${ }^{5}$ Department of Biochemistry, Faculty of Pharmaceutical Sciences in Sosnowiec, Medical University of Silesia in Katowice, Katowice, Poland

${ }^{6}$ Diagnostic Imaging and Interventional Radiology Center, The Independent Public Clinical Hospital No. 6, Medical University of Silesia in Katowice, Katowice, Poland ${ }^{7}$ Department of Pediatric Neurology, Faculty of Medical Sciences, Medical University of Silesia in Katowice, Katowice, Poland

${ }^{8}$ Department of Pathomorphology and Molecular Diagnostics, Medical University of Silesia in Katowice, Katowice, Poland

Institute of Cardiology, Jagiellonian University Medical College, Kraków, Poland

${ }^{10}$ John Paul II Hospital, Kraków, Poland
\end{abstract}

\author{
Correspondence to: \\ Prof. Lesław Szydłowski, MD, \\ $\mathrm{PhD}$, \\ Department of Pediatric \\ Cardiology, Faculty of Medical \\ Sciences, \\ Medical University of Silesia \\ in Katowice, \\ Medyków 16, 40-752 Katowice, \\ Poland, \\ phone: +48 322071855 \\ e-mail: szydlowskil@interia.pl \\ Copyright by the Author(s), 2022 \\ DOI: 10.33963/KP.a2021.0084 \\ Received: \\ July 7, 2021 \\ Accepted: \\ August 6, 2021 \\ Early publication date: \\ August 6, 2021
}

A healthy 14-year-old boy felt unwell while swimming. Soon, he experienced nystagmus. The next day, when he was at the emergency department, he was admitted to the infectious ward since he tested positive in a SARS-CoV-2 antigen test. On admission, abnormal eye movements were observed, double vision when looking to the left, and slight asymmetry of the pupils otherwise without focal features of central nervous system damage. The head computed tomography (CT) scan showed normal cerebral structures. At the same time, brain magnetic resonance imaging $(\mathrm{MRI})$ revealed abnormal intensity in the medial part of the left thalamus corresponding to the acute phase of ischemia (Figure 1A).

The diagnosis of ischemic stroke due to COVID-19-associated coagulopathy was established, and acetylsalicylic acid (ASA) was administered. Assessment of stroke risk factors revealed the homozygosity for the methylenetetrahydrofolate reductase $677 \mathrm{C}>\mathrm{T}$ mutation, while Leiden mutation was not found. The antiphospholipid syndrome was excluded, and other results (homocysteine, protein C, protein S, D-dimer, troponin levels) were normal.

The control brain MRI after 9 days showed the resolution of ischemia. The patient was discharged in overall good condition, and ASA treatment was maintained. Five weeks later, after a negative SARS-CoV-2 test, he was readmitted for cardiology consultation. Echocardiography revealed two movable echogenic structures: in the left ventricular (LV) apex, approximately $2.5 \times 1.5 \mathrm{~cm}$, and in the left atrium (LA) $2 \times 3 \mathrm{~cm}$ (Figure 1B), along with hypokinesis of the apical region with normal ejection fraction of $70 \%$ calculated using the Simpson method. For this reason, cardiac MRI was performed, which confirmed the presence of two large structures in the LV and the LA and post-infarct foci in the $17^{\text {th }}$ apex segment (Figure 1C). Coronary computed tomography angiogram showed no coronary stenosis (thrombus), including the distal left anterior descending coronary artery (Figure $1 \mathrm{E}$ ), and visualized 2 nonenhancing structures in the LV and the LA. After imaging, 


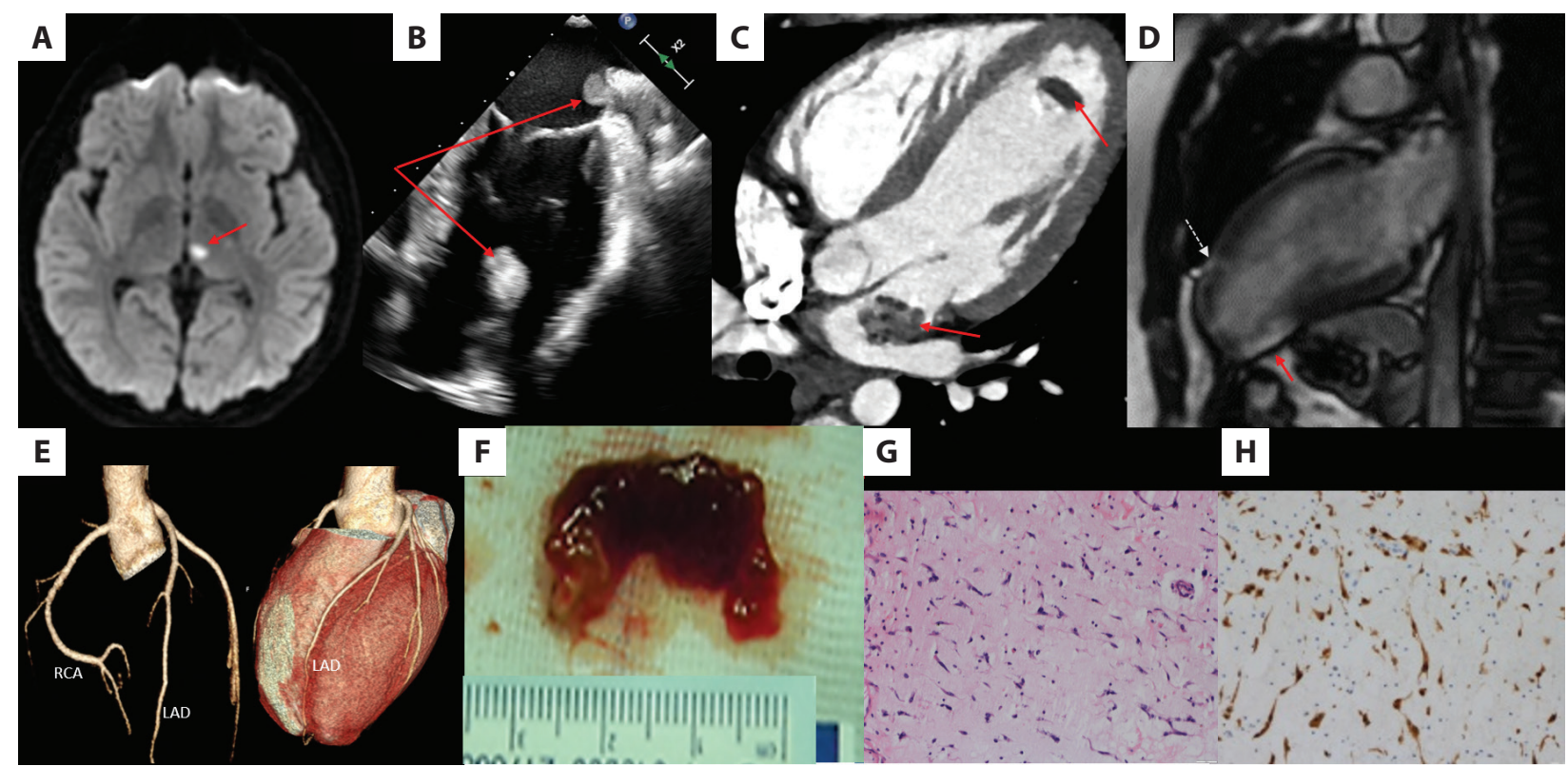

Figure 1. A. Brain MRI scan: axial DWI scan shows the focal region of a restricted diffusion - acute ischemic lesion (arrow) in the left posterior thalamus. B. 2DE image. Two large echogenic structures in the LV and the LA (arrows). C. Cardiac CT, axial view: hypodense soft tissue mass in the LV apex and the LA (arrows) - suspicion of double thrombi on CT. D. Cardiac MRI, the LV 2-chamber view, LGE image: the focus of late gadolinium enhancement in the apical segment of the LV wall (red arrow); the small focus of LGE visible also in the apical segment of the anterior LV wall (white arrow). E. Coronary CTA. 3D reconstruction showing normal coronary arteries. F. anatomical specimen, $2.9 \times 2.1 \mathrm{~cm}$ from the LV apex. G. Myxoma. The mass was composed of stellate and spindle cells embedded within a myxoid stroma (hematoxylin and eosin stain, magnification $\times 100$ ). $\mathbf{H}$. Immunohistochemical staining for calretinin. A markedly positive immunohistochemical reaction in tumor cells. Lymphocytes without calretinin expression (magnification $\times 100$ )

Abbreviations: 2DE, two-dimensional echocardiographic; $\mathrm{CT}$, computed tomography; $\mathrm{CTA}$, computed tomography angiogram; $\mathrm{DWl}$, diffusion weighted; LA, left atrium; LGE, late gadolinium enhancement; LV, left ventricle; MRI, magnetic resonance imaging

the most likely diagnosis was double LV and LA thrombus formation during SARS-CoV-2 infection. Therefore, ASA was discontinued, and nadroparin $2 \times 1 \mathrm{mg} / \mathrm{kg}$ daily s.c. was initiated. After 8 days of intensive treatment, the thrombus did not diminish, and there was a high risk that mobile clots could lead to recurrent stroke. After consultation with several specialists to determine the treatment strategy, we decided to remove the clots during the cardiac surgery (Figure 1F). The result of the histopathology examination was unexpected (Figure 1G, H). It turned out that the masses removed from the heart were double myxomas with clots accumulated on the surface, which were most likely formed during the SARS-CoV-2 infection.

This is a rare case because, among children, myxoma is a rare heart tumor, while dual localization is a unique diagnosis [1-3]. Multiple and recurrent myxomas were rarely described as features of the Carney complex. Although our patient did not exhibit Carney complex symptoms, regular echocardiography follow-up was ordered to prevent myxoma recurrence and potential embolism [4]. Thrombus formation on the surface of cardiac tumors and its complications is a new medical phenomenon during the COVID-19 pandemic [5].

\section{Supplementary material}

Supplementary material is available at https://journals. viamedica.pl/kardiologia_polska.

\section{Article information}

Conflict of interest: None declared.

Open access: This article is available in open access under Creative Common Attribution-Non-Commercial-No Derivatives 4.0 International (CC BY-NC-ND 4.0) license, allowing to download articles and share them with others as long as they credit the authors and the publisher, but without permission to change them in any way or use them commercially. For commercial use, please contact the journal office at kardiologiapolska@ptkardio.pl.

\section{REFERENCES}

1. Morka A, Kohut J, Radzymińska-Chruściel B, et al. Echocardiography and newer imaging techniques in diagnosis and long-term follow-up of primary heart tumors in children. Int J Environ Res Public Health. 2020; 17(15): 5471, doi: 10.3390/ijerph17155471, indexed in Pubmed: 32751243.

2. Colin GC, Gerber BL, Amzulescu M, et al. Cardiac myxoma: a contemporary multimodality imaging review. Int J Cardiovasc Imaging. 2018; 34(11): 1789-1808, doi: 10.1007/s10554-018-1396-z, indexed in Pubmed: 29974293.

3. Velez Torres JM, Martinez Duarte E, Diaz-Perez JA, et al. Cardiac myxoma: review and update of contemporary immunohistochemical markers and molecular pathology. Adv Anat Pathol. 2020; 27(6): 380-384, doi: 10.1097/PAP. 0000000000000275 , indexed in Pubmed: 32732585.

4. Maddali MM, Kandahar PS, Venkatachlam R, et al. Stroke at 4 years unravels a rare diagnosis. J Cardiothorac Vasc Anesth. 2020; 34(6): 1577-1581, doi: 10.1053/j.jvca.2019.11.013, indexed in Pubmed: 31818528.

5. The European Society for Cardiology. ESC Guidance for the Diagnosis and Management of CV Disease during the COVID-19 Pandemic. . https:// www.escardio.org/Education/COVID-19-and-Cardiology/ESC-COVID-19Guidance (July 6, 2021). 